\title{
LOCAL SCALE INVARIANCE AND CONTINGENT CLAIM PRICING II: PATH-DEPENDENT CONTINGENT CLAIMS
}

\author{
J. K. HOOGLAND* and C. D. D. NEUMANN ${ }^{\dagger}$ \\ Centrum voor Wiskunde en Informatica (CWI), \\ P.O. Box 94079, 1090 GB Amsterdam, The Netherlands
}

Received 6 October 1999

Accepted 3 August 2000

\begin{abstract}
This article is the second one in a series on the use of local scale invariance in finance. In the first [6], we introduced a new formalism for the pricing of derivative securities, which focuses on tradable objects only, and which completely avoids the use of martingale techniques. In this article we show the use of the formalism in the context of path-dependent options. We derive compact and intuitive formulae for the prices of a whole range of well-known options such as arithmetic and geometric average options, barriers, rebates and lookback options. Some of these have not appeared in the literature before. For example, we find rather elegant formulae for double barrier options with exponentially moving barriers, continuous dividends and all possible configurations of the barriers. The strength of the formalism reveals itself in the ease with which these prices can be derived. This allowed us to pinpoint some mistakes regarding geometric mean options, which frequently appear in the literature. Furthermore, symmetries such as put-call transformations appear in a natural way within the framework.
\end{abstract}

Keywords: Contingent claim pricing, scale invariance, homogeneity, partial differential equation.

\section{Introduction}

In our previous paper [6] we introduced a new formalism for the pricing of derivative securities, based on the idea of the "relativity" of prices. The core of this formalism is the idea that problems should be formulated in terms of tradable objects only. (Note that we use a broad definition of the term "tradable": every quantity that can be represented by a self-financing portfolio is considered to be a tradable). If this is done, we can show that functions expressing the price of a derivative in terms of prices of the underlying tradables should always be a homogeneous function of degree one. This follows from a simple dimensional analysis argument. The same should therefore be true for payoff functions, in terms of which the contracts are specified. In fact, we claim that any payoff function should be representable by a

*E-mail: jiri@cwi.nl

†E-mail: neumann@cwi.nl 
homogeneous function of degree one in tradables, for else it is ill-defined. In this paper we show that the formalism works very well for path-dependent options, and that this leads to more compact formulae, which can be verified more easily and have a clearer financial interpretation.

Our formulation is based on a PDE approach. We have shown that, by using homogeneity properties, it is very easy to derive a PDE describing the evolution of claim prices without making use of any martingale techniques. The solution of the PDE may, of course, be cast in the form of a Feynman-Kac formula using a Green's function approach. The PDE has an explicit symmetry corresponding to the freedom of choice of a numeraire. The PDE is defined in terms of volatility functions of tradables only, drift terms are irrelevant. The only place where drift terms do play a role is in the analysis of arbitrage: if deterministic relations exist between tradables, there are conditions on the drift terms in order to exclude arbitrage. The fact that our PDE has an explicit numeraire independence is fundamental. It is in marked contrast with the usual Black-Scholes PDE approach. In the latter approach the numeraire, some currency, is fixed in advance and bonds nominated in this currency are considered to be risk-free. This, of course, is an illusion. Even if interest rates are constant, a dollar bond is not risk-free in the eyes of a European investor. In fact, the only object that truly deserves the name "risk-free" is an object that has zero-value at all times. To declare any other object to be risk-free breaks the symmetry and makes calculations a lot less transparent. An important lesson that modern physics teaches us is that symmetries which exist in a problem should be preserved in every step of a calculation. It makes the formalism transparent and gives us a powerful tool to verify results. We want to put forward the opinion that the local scale symmetry is one of the most fundamental ingredients for a pricing theory, and that other concepts, like the existence of an equivalent martingale measure, should be derived from this, not the other way round.

Finally, it should be mentioned that many tricks and symmetries that appear in an ad hoc way in the literature, for example put-call symmetries and similarity reductions, can be traced back to the fundamental property of the homogeneity of pricing functions. It places all these concepts in a unified framework.

The outline of this article is as follows. In Sec. 2 we recall some of the results from Ref. 6. We show how to derive the fundamental PDE using the homogeneity properties of pricing functions, given a set of tradables with stochastic dynamics driven by Brownian motion. Then we show that the PDE posseses a symmetry, associated with numeraire independence, which implies that only the relative volatilities of the underlying tradables with respect to each other matter. Next, we give an algorithm which is used to derive claim prices using this PDE. Furthermore, it is shown that the homogeneity leads in a very straightforward way to generalized put-call symmetries. Finally, we recall the general solution for the case of European claims in a lognormal world, using Green's functions. In Sec. 3 we show that the formulation of the Asian claim pricing problem in terms of the tradables leads to a considerable simplification of the governing PDE. We show that some results regarding 
geometric average options which appear frequently in the literature (e.g. [12]) contain mistakes. We provide the correct solutions. In Sec. 4 we construct a large class of solutions to the PDE, new tradables, which turn out to be very useful in the pricing of barrier-type options in a very compact and intuitive way. In Sec. 5 we discuss the pricing of single and double barrier options, rebates and lookback options. We derive very clean formulae and show the various symmetries between them. We correct and extend some results on double barrier options. We end with a discussion and outlook.

\section{Homogeneity and Contingent Claim Pricing}

In the previous paper [6] we have shown that a fundamental property of any properly defined market of tradables ${ }^{a}$ is that the price of any claim depending on other tradables in the market should be a homogeneous ${ }^{b}$ function of degree one of these same tradables. This property is nothing but a consequence of the simple fact that prices of tradables are only defined with respect to each other. Let us review some of the content of Ref. 6. Assume that we have a market of $n+1$ basic tradables with prices $x_{\mu}(\mu=0, \ldots, n)$ at time $t$. The price of any tradable in this market with a payoff depending on the prices of these basic tradables should satisfy the following scaling symmetry:

$$
V(\lambda x, t)=\lambda V(x, t)
$$

which automatically implies ${ }^{c}$ (Euler)

$$
V(x, t)=x_{\mu} \partial_{x_{\mu}} V(x, t)
$$

where $\partial / \partial x_{\mu} \equiv \partial_{x_{\mu}}$. This is a universal property, independent of the choice of dynamics. We use this fundamental property to derive a general PDE, giving the price of such a claim in a world where the dynamics of the tradables are driven by $k$ independent standard Brownian motions, as follows ${ }^{\mathrm{d}}$

$$
d x_{\mu}(t)=x_{\mu}(t)\left(\sigma_{\mu}(x, t) \cdot d W(t)+\alpha_{\mu}(x, t) d t\right), \quad \text { (no sum) } .
$$

Consistency requires that both $\sigma_{\mu}$ and $\alpha_{\mu}$ are homogeneous functions of degree zero in the tradables, i.e. they should only depend on ratios of prices of tradables. Note that we do not specify the numeraire in terms which the drift and volatility are

\footnotetext{
aTradables are objects which are trivially self-financing: it doesn't cost nor yield money to keep a fixed amount of them. Two examples are stocks and bonds. Note that money is not a tradable, unless the interest rate is zero.

${ }^{\mathrm{b}} \mathrm{A}$ function $f\left(x_{0}, \ldots, x_{n}\right)$ is called homogeneous of degree $r$ if $f\left(a x_{0}, \ldots, a x_{n}\right)=$ $a^{r} f\left(x_{0}, \ldots, x_{n}\right)$. Homogeneous functions of degree $r$ satisfy the following property (Euler): $\sum_{\mu=0}^{n} x_{\mu} \frac{\partial}{\partial x_{\mu}} f\left(x_{0}, \ldots, x_{n}\right)=r f\left(x_{0}, \ldots, x_{n}\right)$

'We make use of Einstein's summation convention: repeated indices in products are implicitly summed over unless otherwise stated.

d Both the $\sigma_{\mu}$ and $d W$ are vectors, the dot denotes an inner-product w.r.t. the $k$ driving diffusions.
} 
expressed. This choice is irrelevant for the pricing problem, as we will see. Applying Itô to $V(x, t)$ we get

$$
d V(x, t)=\partial_{x_{\mu}} V(x, t) d x_{\mu}+\mathcal{L} V(x, t) d t
$$

where

$$
\mathcal{L} V(x, t) \equiv\left(\partial_{t}+\frac{1}{2} \sigma_{\mu}(x, t) \cdot \sigma_{\nu}(x, t) x_{\mu} x_{\nu} \partial_{x_{\mu}} \partial_{x_{\nu}}\right) V .
$$

So, if $V(x, t)$ solves $\mathcal{L} V=0$ with the payoff at maturity as the boundary condition $V(x, T)=f(x)$, we immediately have a replicating self-financing trading strategy because of the homogeneity property. We will drop the distinction between such derived and basic quantities and always refer to them as tradables. Note that we do not have to use any change of measure to arrive at this result if we keep the symmetry explicit. Drifts are irrelevant for the derivation of the claim price. Only the requirement of uniqueness of the solution, i.e. no arbitrage, leads to constraints on the drifts terms if deterministic relations exist between the various tradables [6].

\subsection{Symmetries of the $P D E$}

The scale invariance of the claim price is inherited by the PDE via an invariance of the solutions of the PDE under a simultaneous shift of all volatility functions by an arbitrary function $\lambda(x, t)$

$$
\sigma_{\mu}(x, t) \rightarrow \sigma_{\mu}(x, t)-\lambda(x, t) .
$$

Indeed, if $V$ solves $\mathcal{L} V=0$, then it also solves

$$
\left(\partial_{t}+\frac{1}{2}\left(\sigma_{\mu}(x, t)-\lambda(x, t)\right) \cdot\left(\sigma_{\nu}(x, t)-\lambda(x, t)\right) x_{\mu} x_{\nu} \partial_{x_{\mu}} \partial_{x_{\nu}}\right) V=0 .
$$

This can easily be checked by noting that for homogeneous functions of degree 1 we have

$$
x_{\mu} \partial_{x_{\mu}} \partial_{x_{\nu}} V=0 .
$$

This ensures that terms involving the $\lambda$ drop out of the PDE. (Note that this equation gives interesting relations between the various $\Gamma$ 's of the claim). From this it follows that $V$ itself must be invariant under the substitution defined by Eq. (2.6). This corresponds to the freedom of choice of a numeraire. It just states that volatility is a relative concept. Price functions should not depend on the choice of a numeraire.

\subsection{The algorithm}

To price contingent claims we start out with a basic set of tradables. Using these tradables we may construct new, derived, tradables, whose price-process $V$ depends upon the basic tradables. Of course, these new tradables should be solutions to the 
basic PDE, $\mathcal{L} V=0$. Their payoff functions serve as boundary conditions. (Note that prices of basic tradables trivially satisfy the PDE, by construction). If the derived tradables are constructed in this way, we can use them just like any other tradable. In particular, we can use them as underlying tradables, in terms of which the price of yet other derivative claims can be expressed (and so on ...) In fact, this is a fundamental property that any correctly defined market should posses. It amounts to a proper choice of coordinates to describe the economy.

The general approach to the pricing of a path-dependent claim in our formalism can be described as follows.

(1) The payoff is written in terms of tradable objects.

(2) A PDE is derived for the claim price with respect to these tradables.

(3) The PDE is solved.

(4) Possible consistency check: the solution should be invariant under the substitution Eq. (2.6) (numeraire independence).

\subsection{Generalized put-call symmetries}

As an example of the strength of this symmetry, and to show the natural embedding in our formalism, consider an economy with two tradables with prices denoted by $x_{1,2}$ and dynamics given by $(i=1,2)$

$$
d x_{i}=\sigma_{i}\left(x_{1}, x_{2}, t\right) x_{i} \cdot d W+\cdots
$$

It is easy to see that under certain conditions there should be a generalized putcall symmetry. Any claim with payoff $f\left(x_{1}, x_{2}\right)$ at maturity and price $V\left(x_{1}, x_{2}, t\right)$ should satisfy

$$
\left(\partial_{t}+\frac{1}{2}\left|\sigma\left(x_{1}, x_{2}, t\right)\right|^{2} x_{1}^{2} \partial_{x_{1}}^{2}\right) V=0
$$

where $\sigma\left(x_{1}, x_{2}, t\right)=\sigma_{1}\left(x_{1}, x_{2}, t\right)-\sigma_{2}\left(x_{1}, x_{2}, t\right)$. Homogeneity implies that it also solves

$$
\left(\partial_{t}+\frac{1}{2}\left|\sigma\left(x_{1}, x_{2}, t\right)\right|^{2} x_{2}^{2} \partial_{x_{2}}^{2}\right) V=0
$$

Therefore, if $\left|\sigma\left(x_{1}, x_{2}, t\right)\right|^{2}=\left|\sigma\left(x_{2}, x_{1}, t\right)\right|^{2}$, this PDE can be rewritten as

$$
\left(\partial_{t}+\frac{1}{2}\left|\sigma\left(x_{2}, x_{1}, t\right)\right|^{2} x_{2}^{2} \partial_{x_{2}}^{2}\right) V=0 .
$$

and we see that $V\left(x_{2}, x_{1}, t\right)$ with payoff $f\left(x_{2}, x_{1}\right)$ is a solution, too. This is nothing but a generalized put-call symmetry. In the first case $x_{2}$ acts as numeraire, in the second case $x_{1}$ takes over this role. The usual put-call symmetry follows if we take a constant $\sigma$ and let $x_{1}, x_{2}$ represent a stock and a bond respectively. This result holds also when early-exercise features are included, but extra care should be taken in that case to make sure that the boundary conditions satisfy the symmetry operation. See for example Ref. 2. Furthermore, we would like to point out that in 
the usual formulation, which uses martingales, these symmetries also appear, but only after a lot of work.

\subsection{Lognormal asset prices}

In an economy with lognormal distributed asset prices

$$
d x_{\mu}(t)=\sigma_{\mu}(t) x_{\mu}(t) \cdot d W(t)+\cdots
$$

it is possible to write down a very elegant formula for European-type claims, as was shown in Ref. 6

$$
V\left(x_{0}, \ldots, x_{n}, t\right)=\int V\left(x_{0} \phi\left(z-\theta_{0}\right), \ldots, x_{n} \phi\left(z-\theta_{n}\right), T\right) d^{m} z
$$

with

$$
\phi(z) \equiv \frac{1}{(\sqrt{2 \pi})^{m}} \exp \left(-\frac{1}{2} \sum_{i=1}^{m} z_{i}^{2}\right) .
$$

The $\theta_{\mu}$ are $m$-dimensional vectors, which follow from a singular value decomposition of the covariance matrix $\Sigma_{\mu \nu}$ of rank $m \leq k$ :

$$
\Sigma_{\mu \nu} \equiv \int_{t}^{T} \sigma_{\mu}(u) \cdot \sigma_{\nu}(u) d u=\theta_{\mu} \cdot \theta_{\nu}
$$

\section{Asians}

Asian contingent claims provide payoffs which involve average prices of one (or more) of the underlying tradables. The averaging makes them strongly pathdependent [11], as knowledge of the price path is required for the determination of the average. In this section we discuss arithmetic and geometric average options [10]. We show that by working with tradables it is possible to write down compact PDE's for the price process. For geometric average strike options it is shown that certain results, which appear frequently in the literature (e.g. [12]), contain mistakes.

\subsection{Arithmetic average options}

A general rule is that payoff functions can be expressed as homogeneous functions of degree 1 in tradables. How do we handle information from the past in this context? Let us consider an elementary example, a contract that pays $S(s)$ at time $T>s$. A problem now becomes apparent. How do we relate the value of an object at two different times? A dollar today is not a dollar tomorrow. So we have to define how to translate value through time. What usually is done is to express the value of the stock at time $s$ in a reference currency (say dollars). Since this is a ratio, it is well-defined. This is then converted back into dollars at time $T$. In a formula

$$
S(s) \rightarrow \frac{S(s) P(T, T)}{P(s, s)} .
$$

Here $P(t, T)$ is a bond which pays 1 dollar at time $T$. The next question is: what tradable has this value at time $T$ ? We have to distinguish two time ranges, $t>s$ 
where we know the ratio $\delta \equiv S(s) / P(s, s)$ and $t<s$ where we do not. In the former case, the tradable is simply $\delta P(t, T)$. In the latter case we can consider it as an contract whose value at time $s$ equals $\delta P(s, T)$. Assuming a lognormal world, it has value

$$
\int \frac{S(t) \phi\left(z-\theta_{S}\right) P(t, T) \phi\left(z-\theta_{P_{T}}\right)}{P(t, s) \phi\left(z-\theta_{P_{s}}\right)} d z .
$$

Here we used Eq. (2.14). Therefore, the tradable that should appear in the payoff is given by

$$
V_{S(s)}(t)=\left\{\begin{array}{ll}
\delta P(t, T) & s<t \\
\frac{S(t) P(t, T)}{P(t, s)} \exp \left(\theta_{S}-\theta_{P_{s}}\right) \cdot\left(\theta_{P_{T}}-\theta_{P_{s}}\right) & s>t
\end{array} .\right.
$$

This tradable is an elementary building block for many path-dependent options. For example, an arithmetic average price call option, sampled at discrete times $t_{i}$ $(1 \leq i \leq n)$, has a payoff defined by

$$
\left(\frac{1}{n} \sum_{i=1}^{n} V_{S\left(t_{i}\right)}(T)-K P_{T}\right)^{+}
$$

Note that this formulation allows for stochastic interest rates. To hedge the claim, we need not only the stock $S$ and the bond $P_{T} \equiv P(t, T)$ maturing at time $T$, but also as many bonds as there are sample dates. Obviously, when we consider a continuously sampled average, this becomes problematic. So let us consider a simplification, assuming a deterministic relation between the bond prices

$$
P(t, s)=e^{r(T-s)} P(t, T) .
$$

We will call this a deterministic bond structure. It corresponds to the assumption that interest rates are constant. The bonds do not have to be deterministic, the deterministic relations only imply that all bonds have the same volatility. In this case the currency $N$ in which the bond is nominated can be expressed as

$$
N(t)=P(t, t)=e^{r(T-t)} P(t, T) .
$$

This corresponds to the usual assumptions in the literature when the bonds are deterministic. Now $V_{S(s)}$ reduces to

$$
V_{S(s)}(t)=\left\{\begin{array}{ll}
\delta P(t, T) & s<t \\
e^{-r(T-s)} S(t) & s>t
\end{array} .\right.
$$

Now let us consider options involving a continuously sampled arithmetic average price. This price is represented by the tradable

$$
\begin{aligned}
\bar{S}(t)= & \frac{1}{T} \int_{0}^{T} V_{S(s)}(t) d s=\frac{1-e^{-r(T-t)}}{r T} S(t) \\
& +\frac{1}{T} \int_{0}^{t} \frac{S(s)}{P(s, s)} d s P(t, T) \equiv \phi S+A P .
\end{aligned}
$$


If we take the bond as the numeraire, then

$$
d S=\sigma S d W+\cdots, \quad d \bar{S}=\phi \sigma S d W+\cdots .
$$

If we choose the bond as the numeraire, in this gauge the relative volatility of the bonds is zero. This immediately leads to the following PDE for the price of an arithmetic average option

$$
\left(\partial_{t}+\frac{1}{2} \sigma^{2} S^{2}\left(\partial_{S}+\phi \partial_{\bar{S}}\right)^{2}\right) V=0
$$

Note that if we have a contract that has a payoff, which only depends on $S$ and $\bar{S}$, such as an average strike option, we can further reduce the dimension of the PDE by choosing $S$ as a numeraire. The PDE then becomes

$$
\left(\partial_{t}+\frac{1}{2} \sigma^{2}(\bar{S}-\phi S)^{2} \partial_{\bar{S}}^{2}\right) V=0
$$

Introducing the variable $x=\bar{S} / S$, one sees that the dimension of the PDE is reduced by one. This form can be used as a starting point to find a semi-analytical or numerical solution. We provide more details on semi-analytic solutions in Ref. [7], where we also show that the local scale invariance allows one to relate unseasoned arithmetic average strike and average price options. In Ref. 8 we introduce a very fast scheme to solve the above PDE. Finally, note that if we perform a change of variables in Eq. (3.10) using the running average $A$ instead of $\bar{S}$, we find

$$
\left(\partial_{t}+\frac{S}{N(t) T} \partial_{A}+\frac{1}{2} \sigma^{2} S^{2} \partial_{S}^{2}\right) V=0
$$

which corresponds to the usual formulation.

\subsection{Geometric average options}

What about geometric average options? For these, we need another building block: a tradable paying at time $T$

$$
\log \left(\frac{S(s)}{P(s, s)}\right) P(T, T) .
$$

Again we distinguish two time ranges. For $t>s$, the tradable is $\delta P(t, T)$ where $\delta \equiv \log (S(s) / P(s, s))$ is known. For $t<s$ its value is given by

$$
\int \log \left(\frac{S(t) \phi\left(z-\theta_{S}\right)}{P(t, s) \phi\left(z-\theta_{P_{s}}\right)}\right) P(t, T) \phi\left(z-\theta_{P_{T}}\right) d z .
$$

This integral can be evaluated easily, giving

$$
P(t, T)\left(\log \left(\frac{S(t)}{P(t, s)}\right)-\frac{1}{2}\left|\theta_{S}-\theta_{P_{T}}\right|^{2}+\frac{1}{2}\left|\theta_{P_{s}}-\theta_{P_{T}}\right|^{2}\right) .
$$


In the deterministic bond setting, things simplify to

$$
V_{\log (S(s))}(t)=\left\{\begin{array}{ll}
\delta P(t, T) & s<t \\
\left(\log \left(\frac{S(t)}{P(t, T)}\right)-r(T-s)-\frac{1}{2} \sigma^{2}(s-t)\right) P(t, T) & s>t
\end{array} .\right.
$$

(Note: for $s=T$ this is a log-option). From this we can construct a tradable representing the logarithm of the geometric average (up to a factor $T^{-1}$ ):

$$
\begin{aligned}
\bar{s}(t)= & \int_{0}^{T} V_{\log (S(s))}(t) d s \\
= & P(t, T)\left((T-t) \log \left(\frac{S(t)}{P(t, T)}\right)-\frac{1}{2}\left(r+\frac{1}{2} \sigma^{2}\right)(T-t)^{2}\right) \\
& +P(t, T) \int_{0}^{t} \log \left(\frac{S(s)}{P(s, s)}\right) d s .
\end{aligned}
$$

If we take the bond as the numeraire, then

$$
d S=\sigma S d W+\cdots, \quad d \bar{s}=\sigma(T-t) P d W+\cdots ;
$$

Therefore, we find the following PDE for claim prices

$$
\left(\partial_{t}+\frac{1}{2} \sigma^{2}\left(S^{2} \partial_{S}^{2}+2(T-t) S P \partial_{S} \partial_{\bar{s}}+(T-t)^{2} P^{2} \partial_{\bar{s}}^{2}\right)\right) V=0 .
$$

Now it is useful to perform a change of variables. We want to trade in $\bar{s}$ for the following object, which is again a tradable, and equals the geometric average on expiry

$$
\begin{aligned}
\tilde{S} & =P \exp \left(\frac{\bar{s}}{P T}+\frac{\sigma^{2}(T-t)^{3}}{6 T^{2}}\right) \\
& =P \exp \left(\frac{1}{T}\left((T-t) \log \left(\frac{S}{P}\right)-\frac{1}{2}\left(r+\frac{1}{2} \sigma^{2}\right)(T-t)^{2}+I\right)+\frac{\sigma^{2}(T-t)^{3}}{6 T^{2}}\right),
\end{aligned}
$$

where we have introduced $I$ for the integral over $\log (S / P)$. In terms of this tradable, the PDE becomes

$$
\left(\partial_{t}+\frac{1}{2} \sigma^{2}\left(S^{2} \partial_{S}^{2}+2 \frac{T-t}{T} S \tilde{S} \partial_{S} \partial_{\tilde{S}}+\frac{(T-t)^{2}}{T^{2}} \tilde{S}^{2} \partial_{\tilde{S}}^{2}\right)\right) V=0 .
$$

It turns out that the prices we obtain for geometric average options differ from those in the literature. What seems to be missing there is the last term in the exponential in Eq. (3.20). We are convinced that our results are correct. To prove this, let us explicitly show that $\tilde{S}$ satisfies the fundamental PDE

$$
\mathcal{L} \tilde{S}(S, P, t)=\frac{\tilde{S}}{T}\left(\partial_{t} I-\log \left(\frac{S}{P e^{r(T-t)}}\right)\right) .
$$


This vanishes by definition of $I$. Note that the delta's corresponding to $\tilde{S}$ are simple

$$
\partial_{S} \tilde{S}=\left(\frac{T-t}{T}\right) \frac{\tilde{S}}{S}, \quad \partial_{P} \tilde{S}=\left(\frac{t}{T}\right) \frac{\tilde{S}}{P} .
$$

Consider an average price call. Since its payoff $(\tilde{S}-K P)^{+}$only depends on $\tilde{S}$ and $P$, the relevant $\mathrm{PDE}$ reduces to

$$
\left(\partial_{t}+\frac{1}{2} \sigma^{2} \frac{(T-t)^{2}}{T^{2}} \tilde{S}^{2} \partial_{\tilde{S}}^{2}\right) V=0
$$

The solution of this problem is

$$
V_{G A P C}(S, P, K, t)=\tilde{S} \Phi\left(d_{1}\right)-K P \Phi\left(d_{2}\right)
$$

with

$$
d_{1,2} \equiv \frac{\log \left(\frac{\tilde{S}}{K P}\right)}{\Sigma} \pm \frac{1}{2} \Sigma, \quad \Sigma^{2} \equiv \frac{\sigma^{2}(T-t)^{3}}{3 T^{2}} .
$$

Here $\Phi(z) \equiv \int_{-\infty}^{z} \exp \left(-\frac{1}{2} x^{2}\right) d x / \sqrt{2 \pi}$. If we look at the price of the option at $t=0$, and use the currency corresponding to the bond as the numeraire (so that $P=$ $\left.e^{-r(T-t)}\right)$, this formula reduces to

$$
\begin{aligned}
& V_{G A P C}(S, P, K, 0) \\
& \quad=e^{-a T} S \Phi\left(\frac{\log \left(\frac{S}{K}\right)+a T}{\Sigma}\right)-e^{-r T} K \Phi\left(\frac{\log \left(\frac{S}{K}\right)+a T}{\Sigma}-\Sigma\right) .
\end{aligned}
$$

Here $a=\frac{1}{2}\left(r+\frac{\sigma^{2}}{6}\right)$. We wrote this in the format used in [12], but found a different result. Next consider an average strike call. After a suitable change of numeraire, the PDE becomes

$$
\left(\partial_{t}+\frac{1}{2} \sigma^{2} \frac{t^{2}}{T^{2}} S^{2} \partial_{S}^{2}\right) V=0
$$

In this case, the solution is

$$
V_{G A S C}(S, \tilde{S}, t)=S \Phi\left(d_{1}\right)-\tilde{S} \Phi\left(d_{2}\right)
$$

with

$$
d_{1,2} \equiv \frac{\log \left(\frac{S}{\tilde{S}}\right)}{\Sigma} \pm \frac{1}{2} \Sigma, \quad \Sigma^{2} \equiv \frac{\sigma^{2}\left(T^{3}-t^{3}\right)}{3 T^{2}} .
$$

Again, if we look at the price of the option at $t=0$, and use the currency corresponding to the bond as the numeraire this formula reduces to

$$
V_{G A S C}(S, \tilde{S}, 0)=S \Phi\left(\frac{a T}{\Sigma}+\frac{1}{2} \Sigma\right)-e^{-a T} S \Phi\left(\frac{a T}{\Sigma}-\frac{1}{2} \Sigma\right) .
$$




\section{Generating New Solutions}

In this section we consider claims depending on one lognormal stock $S$ with volatility $\sigma$ in a deterministic bond structure as before. It should be clear that in our approach it is a trivial exercise to write down the corresponding formula, when we have two lognormal stocks instead of one stock and one bond. We show that the symmetries of the PDE allow for the construction of classes of solutions, which prove very useful in constructing solutions for barrier-type claims. The governing PDE for the claim-price $V(S, P, t)$ with payoff $f(S, P)$ at time $T$ can be written as

$$
\mathcal{L} V \equiv\left(\partial_{t}+\frac{1}{2} \sigma^{2} S^{2} \partial_{S}^{2}\right) V=0 .
$$

In the following we will use time-to-maturity $\tau \equiv T-t$ instead of the running time $t$ to simplify the notation. The PDE becomes

$$
\mathcal{L} V \equiv\left(-\partial_{\tau}+\frac{1}{2} \sigma^{2} S^{2} \partial_{S}^{2}\right) V=0 .
$$

The Green's function for this PDE is given by

$$
G(x, \tau)=\frac{1}{\Sigma} \phi\left(\frac{\log (x)}{\Sigma}-\frac{1}{2} \Sigma\right), \quad \Sigma \equiv \sigma \sqrt{\tau}, \quad \phi(z) \equiv \frac{1}{\sqrt{2 \pi}} e^{-\frac{1}{2} z^{2}} .
$$

Now we construct a tradable $R_{\alpha}(S, P, \tau)$ with payoff $F_{\alpha}(S, P) \equiv(S / P)^{\alpha} P$ at maturity for some constant $\alpha$. The convolution of the payoff function with the Green's function yields a new set of tradables

$$
R_{\alpha}(S, P, \tau) \equiv\left(\frac{S}{e^{-\frac{1}{2} \sigma^{2} \alpha t} P}\right)^{\alpha} e^{-\frac{1}{2} \sigma^{2} \alpha \tau} P .
$$

Recall that a tradable is a trivially self-financing object. Note that $R_{0}(S, P, \tau)=P$ and $R_{1}(S, P, \tau)=S$. These tradables satisfy two very nice symmetry properties. On the one hand we have

$$
R_{\alpha}(S, P, \tau)=R_{-\alpha+1}(P, S, \tau)
$$

On the other hand

$$
\begin{gathered}
R_{\alpha}\left(R_{\alpha}(S, P, \tau), R_{\alpha+1}(S, P, \tau), \tau\right)=S \\
R_{\alpha+1}\left(R_{\alpha}(S, P, \tau), R_{\alpha+1}(S, P, \tau), \tau\right)=P .
\end{gathered}
$$

These are special cases of the following relations

$$
\begin{aligned}
& R_{\alpha}\left(R_{\beta}(S, P, \tau), R_{\beta+1}(S, P, \tau), \tau\right)=R_{\beta-\alpha+1}(S, P, \tau) \\
& R_{\alpha}\left(R_{\beta+1}(S, P, \tau), R_{\beta}(S, P, \tau), \tau\right)=R_{\beta+\alpha}(S, P, \tau) .
\end{aligned}
$$

If we apply Itô to $R_{\alpha}(S, P, \tau)$ we obtain

$$
d R_{\alpha}=\alpha \sigma R_{\alpha} d W+\cdots
$$


Therefore the price $V\left(\lambda R_{\alpha}, \mu R_{\beta}, \tau\right)$ of a claim with payoff $f\left(\lambda R_{\alpha}, \mu R_{\beta}\right)$ at maturity with $\lambda, \mu \in \mathbf{R}$ satisfies the following PDE:

$$
\left(-\partial_{\tau}+\frac{1}{2}(\alpha-\beta)^{2} \sigma^{2} R_{\alpha}^{2} \partial_{R_{\alpha}}^{2}\right) V=0 .
$$

If we introduce a scaled time $\tilde{\tau} \equiv(\alpha-\beta)^{2} \tau$ this PDE becomes of the form

$$
\left(-\partial_{\tilde{\tau}}+\frac{1}{2} \sigma^{2} R_{\alpha}^{2} \partial_{R_{\alpha}}^{2}\right) V=0
$$

which bears close resemblance to the PDE at the beginning of this section. This implies that if $V(S, P, \tau)$ solves $\mathcal{L} V=0$ with the payoff $V(S, P, 0)=f(S, P)$ as boundary-condition, then

$$
V\left(\lambda R_{\alpha}, \mu R_{\beta},(\alpha-\beta)^{2} \tau\right)
$$

is a solution too of the PDE with payoff $f\left(\lambda R_{\alpha}, \mu R_{\beta}, 0\right)$. Let us consider a few simple examples. If we denote by $V_{C}(S, P, \tau)$ the price of a vanilla call with strike 1 and payoff $f_{C}(S, P)=(S-P)^{+}$is then

$$
V_{C}(S, K P, \tau)=V_{C}\left(R_{1}, K R_{0}, \tau\right)
$$

with strike $K$ will be a solution with payoff $f_{C}(S, K P)$. In a similar way we define the price $V_{P}(S, P, \tau)$ of a vanilla put with strike 1 and payoff $f_{P}(S, P)=(P-S)^{+}$. Automatically $V_{P}(S, K P, \tau)$ will be a solution, too, with payoff $f_{P}(S, K P)$. The put-call parity transformation follows immediately:

$$
V_{C}(S, K P, \tau)=V_{P}(K P, S, \tau) .
$$

It also follows trivially that when $K_{C} K_{P}=(S / P)^{2}$,

$$
V_{C}\left(S, K_{C} P, \tau\right)=\sqrt{K_{C} / K_{P}} V_{P}\left(S, K_{P} P, \tau\right) .
$$

Note that $S / P$ is what is called the forward price in the literature.

Finally, note that we may construct additional solutions to a PDE with a time-dependent volatility-function $\sigma(\tau)$. In this case the solutions are of the form $V\left(\lambda R_{\alpha}, \mu R_{\alpha \pm 1}, \tau\right)$ where

$$
R_{\alpha}(S, P, \tau)=\left(\frac{S}{e^{\frac{1}{2} \Sigma^{2} \alpha} P}\right)^{\alpha} e^{\frac{1}{2} \Sigma^{2} \alpha} P
$$

and $\Sigma^{2} \equiv \int_{0}^{T-t} \sigma(u)^{2} d u$.

The earlier quoted symmetries can be used to relate the various solutions of the PDE and can be used very fruitfully in the construction of solutions of, for example, barrier-type options. These symmetries lead to generalized put-call symmetries, although they also provide symmetries between vanilla and barrier options, as we will show in the next section.

These types of symmetries may be very useful when one needs to hedge an exotic contingent claim, as was already observed in Ref. 3. Especially with barrier-type options it allows one to introduce semi-static hedges. 


\section{Barriers and Friends}

In this section we discuss contingent claims which possess so-called "weak path dependence" [11]. These claims have payoff features which are triggered by some event during the life-time of the contract. In contrast to the asian-type options, which are strongly path-dependent, their valuation is less involved, as it does not require knowledge of the complete path. We first consider single moving barriers, where we show the usefulness of the symmetry of solutions discussed in the previous section. Then we move on to double moving barriers claims, which can be seen as a double infinite sum of single moving barriers claims. Only a few terms are required to obtain accurate results. We show that the results of Ref. 9 are valid only when $L<K<U$, where $L$ and $U$ denote the lower- and upper-barrier and $K$ the strike, and give the correct results for the general case including continuous

dividends. Finally, we discuss lookback options and show that they fit nicely into our framework.

Note that barriers are often monitored at discrete times. In Ref. 1 a simple and straightforward way to correct for this fact has been put forward. It only involves a simple shift of the barriers depending on the frequency of monitoring.

\subsection{Single barriers}

We start our discussion with the simplest type of a single barrier claim, a down-andout call. This is a call option with the additional feature that it becomes worthless when the stockprice hits a barrier, given by $S=B e^{\gamma \tau} P \equiv B(\tau) P$, from above during the lifetime of the option. Here we have $B, \gamma \in \mathbf{R}$. As before, we assume that we have a deterministic bond structure. This implies that $\gamma=r$ corresponds to a constant barrier (in terms of the currency in which the bond is nominated), while other values of $\gamma$ lead to exponentially moving barriers. The claim price should satisfy the standard PDE

$$
\left(-\partial_{\tau}+\frac{1}{2} \sigma^{2} S^{2} \partial_{S}^{2}\right) V_{D O C}(S, P, K, B, \gamma, \tau)=0
$$

It is well known that all specific properties of this option are in the boundary conditions. The boundary condition for the European down-and-out call are simply

$$
\begin{array}{cc}
V_{D O C}(S, P, K, B, \gamma, 0)=(S-K P)^{+} & \text {for all } S>B P \\
V_{D O C}(B(\tau) P, P, K, B, \gamma, \tau)=0 & \text { for all } \tau>0 .
\end{array}
$$

We will now assume that $B<K$, and come back to the case $B>K$ later. Let us first consider the case where we do not have the second boundary condition and the payoff is defined on the whole positive $S$-axis. This is just the standard European call $V_{C}(S, K P, \tau)$. The second boundary condition says that the value of the option becomes zero if, during its lifetime, the barrier is hit. This implies that the option price should be lower than that of a plain call since we take more risk. Thus we have to subtract a barrier premium from the standard call price. 
Let us write $\tilde{V}(S, P, K, B, \gamma, \tau)$ for this barrier premium. What boundary conditions should this function obey? Of course, its value should coincide with $V_{C}(S, K P, \tau)$ on the boundary $S=B(\tau) P$ for all $\tau>0$ :

$$
V_{C}(B(\tau) P, K P, \tau)=\tilde{V}(B(\tau) P, P, K, B, \gamma, \tau)
$$

If, in addition, we have $\tilde{V}(S, P, K, B, \gamma, 0)=0$ for $S>B P$, then we can construct the solution to the down-and-out call by

$$
V_{D O C}(S, P, K, B, \gamma, \tau)=V_{C}(S, K P, \tau)-\tilde{V}(S, P, K, B, \gamma, \tau) .
$$

At this point we invoke the results of the previous section. If $V_{C}(S, P, \tau)$ solves $\mathcal{L} V=0$, then so does

$$
\tilde{V}(S, P, K, B, \gamma, \tau)=V_{C}\left(\lambda R_{\alpha}(S, B P, \tau), \mu R_{\beta}(S, B P, \tau),(\alpha-\beta)^{2} \tau\right) .
$$

With this choice of the barrier premium Eq. (5.5) satisfies all boundary conditions if we set

$$
\lambda=1, \quad \mu=\frac{K}{B}, \quad \alpha=-\frac{2 \gamma}{\sigma^{2}}, \quad \beta=\alpha+1 .
$$

The solution is thus given by

$$
\begin{aligned}
& V_{D O C}(S, P, K, B, \gamma, \tau) \\
& \quad=V_{C}(S, K P, \tau)-V_{C}\left(R_{\alpha}(S, B P, \tau), \frac{K}{B} R_{\alpha+1}(S, B P, \tau), \tau\right) .
\end{aligned}
$$

If $B>K$, we can apply the same construction. Only the call $V_{C}$ should now be replaced by a "left-clipped" call $V_{C}^{B+}$, which can be defined by its payoff

$$
V_{C}^{B+}(S, K P, 0)=\left\{\begin{array}{ll}
S-K P & \text { for } S>B P \\
0 & \text { otherwise }
\end{array} .\right.
$$

Thus its value is given by

$$
V_{C}^{B+}(S, K P, \tau)=S \Phi\left(d_{1}\right)-K P \Phi\left(d_{2}\right)
$$

with

$$
d_{1,2}=\frac{\log \left(\frac{S}{B P}\right)}{\Sigma} \pm \frac{1}{2} \Sigma, \quad \Sigma \equiv \sigma \sqrt{\tau} .
$$

In a similar way, we can value an up-and-out call. Such an option is only interesting for $B>K$, since otherwise it is worthless. Repeating the same arguments, we see that it can be defined in terms of a "right-clipped" call $V_{C}^{B-}$, with payoff

$$
V_{C}^{B-}(S, K P, 0)=\left\{\begin{array}{ll}
S-K P & \text { for } K P<S<B P \\
0 & \text { otherwise }
\end{array} .\right.
$$


Its value follows from $V_{C}^{B-}=V_{C}-V_{C}^{B+}$. So the value of the up-and-out call is given by

$$
\begin{aligned}
& V_{U O C}(S, P, K, B, \gamma, \tau) \\
& \quad=V_{C}^{B-}(S, K P, \tau)-V_{C}^{B-}\left(R_{\alpha}(S, B P, \tau), \frac{K}{B} R_{\alpha+1}(S, B P, \tau), \tau\right)
\end{aligned}
$$

The values of up/down-and-in call follow from in-out parity

$$
\begin{aligned}
& V_{D I C}(S, P, K, B, \gamma, \tau)=V_{C}(S, K P, \tau)-V_{D O C}(S, P, K, B, \gamma, \tau) \\
& V_{U I C}(S, P, K, B, \gamma, \tau)=V_{C}(S, K P, \tau)-V_{U O C}(S, P, K, B, \gamma, \tau) .
\end{aligned}
$$

Note that under the transformations $R_{\alpha} \leftrightarrow S, R_{\alpha+1} \leftrightarrow P$ we find a vanilla-barrier transformation which is valid for $B<K$

$$
V_{D I C}\left(R_{\alpha}(S, B P, \tau), \frac{R_{\alpha+1}(S, B P, \tau)}{B}, K, B, \gamma, \tau\right)=V_{C}(S, K P, \tau) .
$$

For a down-and-out call we find a similar symmetry, which is actually valid for all $B$ :

$$
V_{D O C}\left(R_{\alpha}(S, B P, \tau), \frac{R_{\alpha+1}(S, B P, \tau)}{B}, K, B, \gamma, \tau\right)=-V_{D O C}(S, P, K, B, \gamma, \tau) .
$$

And similarly for the up-and-out call. Furthermore, we can immediately write down the price for all single barrier put options by using the generalized put-call transformation

$$
\begin{aligned}
& V_{U O P}(S, P, K, B, \gamma, \tau)=K V_{D O C}\left(P, S, \frac{1}{K}, \frac{1}{B},-\gamma, \tau\right) \\
& V_{U I P}(S, P, K, B, \gamma, \tau)=K V_{D I C}\left(P, S, \frac{1}{K}, \frac{1}{B},-\gamma, \tau\right) \\
& V_{D O P}(S, P, K, B, \gamma, \tau)=K V_{U O C}\left(P, S, \frac{1}{K}, \frac{1}{B},-\gamma, \tau\right) \\
& V_{D I P}(S, P, K, B, \gamma, \tau)=K V_{U I C}\left(P, S, \frac{1}{K}, \frac{1}{B},-\gamma, \tau\right) .
\end{aligned}
$$

It is a simple check to see that these claim prices satisfy all appropriate boundary conditions.

Let us show that the results above can be rewritten in the more well-known form using

$$
\begin{aligned}
R_{\alpha}(S, B P, \tau) & =\left(\frac{S}{e^{-\frac{1}{2} \sigma^{2} \alpha t} B P}\right)^{\alpha} e^{-\frac{1}{2} \sigma^{2} \alpha \tau} B P \\
& =\left(\frac{S}{e^{\gamma \tau} B P}\right)^{\alpha} e^{\gamma \tau} B P=\left(\frac{S}{B(\tau) P}\right)^{\alpha} B(\tau) P
\end{aligned}
$$




$$
\begin{aligned}
R_{\alpha+1}(S, B P, \tau) & =\left(\frac{S}{e^{-\frac{1}{2} \sigma^{2}(\alpha+1) \tau} B P}\right)^{\alpha+1} e^{-\frac{1}{2} \sigma^{2}(\alpha+1) \tau} B P \\
& =\left(\frac{S}{e^{\gamma \tau} B P}\right)^{\alpha} \frac{S}{e^{\gamma \tau}}=\left(\frac{S}{B(\tau) P}\right)^{\alpha} \frac{B S}{B(\tau)} .
\end{aligned}
$$

For example

$$
\begin{aligned}
\tilde{V}(S, P, K, B, \gamma, \tau) & =V_{C}\left(R_{\alpha}(S, B P, \tau), \frac{K}{B} R_{\alpha+1}(S, B P, \tau), \tau\right) \\
& =\left(\frac{S}{B(\tau) P}\right)^{-\frac{2 \gamma}{\sigma^{2}}+1} V_{C}\left(\frac{(B(\tau) P)^{2}}{S}, K P, \tau\right) .
\end{aligned}
$$

The result quoted in the literature corresponds to the case where prices are expressed in the currency. If we consider a constant barrier (setting $\gamma=r$ ), the above equation collapses to

$$
V_{D O C}(S, P, K, B, \gamma, \tau)=V_{C}(S, K P, \tau)-\left(\frac{S}{B}\right)^{-\frac{2 r}{\sigma^{2}}+1} V_{C}\left(\frac{B^{2}}{S}, K P, \tau\right),
$$

as it should.

\subsection{Rebates}

Often, barrier options specify a rebate, an amount of money paid to the holder if the barrier is hit in the case of a knock-out option or not hit in the case of a knockin option. The premium that has to be paid for this provision can be calculated in terms of a rebate option. For example, let us consider a (generalized) down-and-out rebate option ${ }^{\mathrm{e}} V_{D O R}\left(S, P, B, K, \gamma_{1}, \gamma_{2}, \tau\right)$. This option pays the holder $K e^{\gamma_{2} \tau} P$ at the first moment $\tau>0$ for which $S=B e^{\gamma_{1} \tau} P$. The usual choice is $\gamma_{1}=\gamma_{2}=r$, corresponding to a fixed barrier $B$ and a fixed rebate $K$ in terms of money. The option can be characterized by the following boundary conditions:

$$
\begin{aligned}
V_{D O R}\left(B e^{\gamma_{1} \tau} P, P, B, K, \gamma_{1}, \gamma_{2}, \tau\right) & =K e^{\gamma_{2} \tau} P & & \text { for all } \tau>0 \\
V_{D O R}\left(S, P, B, K, \gamma_{1}, \gamma_{2}, 0\right) & =0 & & \text { for all } S>B P .
\end{aligned}
$$

To solve this problem, we first construct a tradable which has the proper value at the boundary

$$
\lambda R_{\alpha}(S, B P, \tau)=K e^{\gamma_{2} \tau} P \text { for } S=B e^{\gamma_{1} \tau} P .
$$

We find that this equation has two solutions, given by

$$
\lambda=\frac{K}{B}, \quad \alpha_{ \pm}=\frac{a \pm b}{\sigma^{2}}, \quad a=\gamma_{1}-\frac{1}{2} \sigma^{2}, \quad b=\sqrt{a^{2}+2 \gamma_{2} \sigma^{2}} .
$$

a.k.a. (generalized) American digital option 
We see that $V_{D O R}-\lambda R_{\alpha_{-}}$should vanish on the boundary. Now we can apply essentially the same techniques as we did for the down-and-out call to find that the rebate option can be written as

$$
\begin{aligned}
V_{D O R} & \left.S, P, B, K, \gamma_{1}, \gamma_{2}, \tau\right) \\
= & \frac{K}{B}\left(R_{\alpha_{-}}(S, B P, \tau)+V_{D}\left(R_{\alpha_{+}}(S, B P, \tau), R_{\alpha_{-}}(S, B P, \tau),\left(\frac{2 b}{\sigma^{2}}\right)^{2} \tau\right)\right. \\
& \left.-V_{D}\left(R_{\alpha_{-}}(S, B P, \tau), R_{\alpha_{+}}(S, B P, \tau),\left(\frac{2 b}{\sigma^{2}}\right)^{2} \tau\right)\right) .
\end{aligned}
$$

Here $V_{D}(S, P, \tau)$ is an asset-or-nothing digital option, which is defined as

$$
V_{D}(S, P, \tau)=S \Phi\left(\frac{\log \left(\frac{S}{P}\right)}{\Sigma}+\frac{1}{2} \Sigma\right), \quad \Sigma \equiv \sigma \sqrt{\tau} .
$$

The up-and-out rebate can be found by using a generalized put-call transformation. It is given by

$$
V_{U O R}\left(S, P, B, K, \gamma_{1}, \gamma_{2}, \tau\right)=V_{D O R}\left(P, S, \frac{1}{B}, \frac{K}{B},-\gamma_{1},-\gamma_{1}+\gamma_{2}, \tau\right) \text {. }
$$

What about knock-in rebates? We cannot simply use in-out parity to find values for these options because of the difference in timing of the payoff. Knock-in rebates are always paid at maturity, while knock-out rebates are generally paid before maturity. However, we can easily calculate the value of a knock-out rebate which does pay out at maturity, simply by setting $\gamma_{2}=0$. This allows us to use in-out parity after all and write

$$
\begin{aligned}
& V_{D I R}\left(S, P, B, K, \gamma_{1}, \tau\right)=K P-V_{D O R}\left(S, P, B, K, \gamma_{1}, 0, \tau\right) \\
& V_{U I R}\left(S, P, B, K, \gamma_{1}, \tau\right)=K P-V_{U O R}\left(S, P, B, K, \gamma_{1}, 0, \tau\right) .
\end{aligned}
$$

\subsection{Double barriers}

Next, let us consider a call option with strike $K$ which knocks out on two boundaries. The upper boundary is defined by $S=H e^{\gamma_{1} \tau} P$, the lower by $S=L e^{\gamma_{2} \tau} P$. Again, the choice $\gamma_{1}=\gamma_{2}=r$ corresponds to constant boundaries. We assume that $L<$ $K<H$. A price for such a call can be constructed in an iterative way. As a first approximation, we consider an up-and-out call, knocking out on the upper boundary

$$
V_{O C}\left(S, P, K, H, L, \gamma_{1}, \gamma_{2}, \tau\right) \sim V_{U O C}\left(S, P, K, H, \gamma_{1}, \tau\right)
$$

Of course, this overestimates the price, since this up-and-out call has a positive value on the lower boundary. To compensate, we add a correction term $V_{1}$ which has the opposite value on this boundary, and which has value zero at maturity if 
$S>L P$. In the same way as before, we find that this correction term can be written as

$$
\begin{aligned}
V_{1} & =\left(\frac{H}{L}\right)^{\frac{2 \gamma_{1}}{\sigma^{2}}+1} V_{U O C}\left(X_{1}, \frac{Y_{1}}{H}, \frac{K L}{H}, L, \gamma_{1}, \tau\right) \\
X_{1} & =R_{\frac{2\left(\gamma_{1}-\gamma_{2}\right)}{\sigma^{2}}+1}(S, L P, \tau) \\
Y_{1} & =R_{\frac{2\left(\gamma_{1}-\gamma_{2}\right)}{\sigma^{2}}}(S, L P, \tau) .
\end{aligned}
$$

But now, the value of the option is not vanishing on the upper boundary. So we need another correction term $V_{-1}$, which compensates this, and has zero value at maturity if $S<H P$. Such a term can be constructed as

$$
\begin{aligned}
& V_{-1}=\left(\frac{H}{L}\right)^{-\frac{2 \gamma_{1}}{\sigma^{2}}-1} V_{U O C}\left(X-1, \frac{Y_{-1}}{H}, \frac{K H}{L}, \frac{H^{2}}{L}, \gamma_{1}, \tau\right) ; \\
& X_{-1}=R_{-\frac{2\left(\gamma_{1}-\gamma_{2}\right)}{\sigma^{2}}+1}\left(S, \frac{H^{2}}{L} P, \tau\right) ; \\
& Y_{-1}=R_{-\frac{2\left(\gamma_{1}-\gamma_{2}\right)}{\sigma^{2}}}\left(S, \frac{H^{2}}{L} P, \tau\right) .
\end{aligned}
$$

Continuing in this way, we find an infinite series representation for the price of the out-call. The successive terms in this sum represent up-and-out calls which are more and more out of the money, so that the corrections terms rapidly become smaller, and in practice we need only a few terms to find a price with reasonable accuracy. The final formula becomes

$$
V_{O C}\left(S, P, K, H, L, \gamma_{1}, \gamma_{2}, \tau\right)=\sum_{n=-\infty}^{\infty} V_{n}
$$

where

$$
\begin{aligned}
& V_{n}=\left(\frac{H}{L}\right)^{n\left(\frac{2 \gamma_{1}}{\sigma^{2}}+1\right)} V_{U O C}\left(X, \frac{Y_{n}}{H}, \frac{K L^{n}}{H^{n}}, \frac{H L^{n}}{H^{n}}, \gamma_{1}, \tau\right) ; \\
& X_{n}=R_{\frac{2 n\left(\gamma_{1}-\gamma_{2}\right)}{\sigma^{2}}+1}\left(S, \frac{H L^{n}}{H^{n}} P, \tau\right) ; \\
& Y_{n}=R_{\frac{2 n\left(\gamma_{1}-\gamma_{2}\right)}{\sigma^{2}}}\left(S, \frac{H L^{n}}{H^{n}} P, \tau\right) .
\end{aligned}
$$

The functions $V_{n}$ have the property that $V_{n}+V_{-n}$ vanishes on the upper boundary and $V_{n}+V_{-n+1}$ vanishes on the lower boundary. Furthermore, $V_{n}=0$ at maturity if $L<S<H$ and $n \neq 0$. We have checked this result against that of [9] and found identical results and rates of convergence. By using in-out parity, we find the following formula for the price of an knock-in call with two barriers

$$
V_{I C}\left(S, P, K, H, L, \gamma_{1}, \gamma_{2}, \tau\right)=V_{U I C}\left(S, P, K, H, \gamma_{1}, \tau\right)-\sum_{n \neq 0} V_{n} .
$$


Generalized put-call transformations give the price for the corresponding double boundary put options

$$
\begin{aligned}
& V_{O P}\left(S, P, K, H, L, \gamma_{1}, \gamma_{2}, \tau\right)=K V_{O C}\left(P, S, \frac{1}{K}, \frac{1}{L}, \frac{1}{H},-\gamma_{2},-\gamma_{1}, \tau\right) \\
& V_{I P}\left(S, P, K, H, L, \gamma_{1}, \gamma_{2}, \tau\right)=K V_{I C}\left(P, S, \frac{1}{K}, \frac{1}{L}, \frac{1}{H},-\gamma_{2},-\gamma_{1}, \tau\right)
\end{aligned}
$$

What will happen when $K<L<H$ in the case of a double barrier call option? In the derivation of the above formulae we have made essential use of the fact that the option-value $V_{U O C}\left(S, P, K, H, \gamma_{1}, 0\right)$ vanishes for $S<L P$. However, this is no longer true when $K<L$. So in order to find a price for this configuration of barriers, we need a new building block, the "left-clipped" up-and-out call. It can be defined as follows:

$$
\begin{aligned}
& V_{U O C}^{c}(S, P, K, H, L, \gamma, \tau) \\
& \quad=V_{C}^{H, L}(S, K P, \tau)-V_{C}^{H, L}\left(R_{\alpha}(S, H P), \frac{K}{H} R_{\alpha+1}(S, H P), \tau\right)
\end{aligned}
$$

where the option $V_{C}^{H, L}$ is defined by the double clipped payoff

$$
V_{C}^{H, L}(S, K P, 0)=\left\{\begin{array}{ll}
S-K P & \text { for } L P<S<H P \\
0 & \text { otherwise }
\end{array} .\right.
$$

Its price is thus given by $V_{C}^{H, L}=V_{C}^{L+}-V_{C}^{H+}$. Now $V_{U O C}^{c}\left(S, P, K, H, L, \gamma_{1}, 0\right)$ does vanish for $S<L P$. Therefore in the case when $K<L<H$, we need to replace the function $V_{n}$ by the following modified functions, a fact which was not recognized in [9]:

$$
\tilde{V}_{n}=\left(\frac{H}{L}\right)^{n\left(\frac{2 \gamma_{1}}{\sigma^{2}}+1\right)} V_{U O C}^{c}\left(X n, \frac{Y_{n}}{H}, \frac{L^{n}}{H^{n}} K, \frac{L^{n}}{H^{n}} H, \frac{L^{n}}{H^{n}} L, \gamma_{1}, \tau\right) .
$$

As a final note, let us mention that continuous dividend payments with rate $q$ can easily be incorporated by making the usual substitution $S \rightarrow S e^{-q \tau}$. Simultaneously, all $\gamma$ 's that appear in the definition of boundaries should be adjusted like $\gamma \rightarrow \gamma-q$.

\subsection{Lookback options}

We now turn to lookback options. Let us consider a floating strike lookback put. It pays the owner the difference between the maximum realized price and the spot price of some asset at expiry. This maximum is usually defined with respect to a given currency. Assuming that we are in a deterministic bond setting, it can be written as

$$
S_{\max }=\max _{0 \leq t \leq T} \frac{S(t)}{e^{\gamma \tau} P(t, T)}
$$


with $\gamma=r$. We will consider a slightly more general definition, leaving $\gamma$ arbitrary. Interestingly, this option can also be described in terms of a boundary problem (Ref. 4). If we introduce $J=S_{\max } P$ and denote the price of the option by $V_{L P}(S, J, \gamma, \tau)$, the boundary conditions are

$$
\begin{aligned}
V_{L P}(S, J, \gamma, 0) & =(J-S)^{+} \\
\left.\partial_{J} V_{L P}(S, J, \gamma, \tau)\right|_{S=e^{\gamma \tau} J} & =0, \quad \text { for all } \tau .
\end{aligned}
$$

The latter condition allows to roll-up the position self-financingly when $S$ reaches a new high. It implies that at the boundary $S=e^{\gamma \tau} J$ all money is invested in the stock. Now let us try a solution of the following form

$$
V_{L P}(S, J, \gamma, \tau)=V_{P}(S, J, \tau)+V_{P}\left(\lambda R_{\alpha}(S, J, \tau), \mu R_{\beta}(S, J, \tau),(\alpha-\beta)^{2} \tau\right),
$$

where $V_{P}(S, J, t)$ is the price of a plain vanilla put with strike $S_{\max }$. One can check that this is indeed a solution, provided that we have

$$
\lambda=\mu=\frac{1}{k}, \quad \alpha=1-k, \quad \beta=1, \quad k=\frac{2 \gamma}{\sigma^{2}} .
$$

Note that we must have $\gamma>0$ or else the price blows up. In greater detail, the solution is

$$
\begin{aligned}
& V_{L P}(S, J, \gamma, \tau) \\
& \quad=J \Phi\left(-d_{2}\right)-S \Phi\left(-d_{1}\right)+\frac{S}{k}\left(\Phi\left(d_{1}\right)-e^{-\gamma \tau}\left(\frac{e^{\gamma \tau} J}{S}\right)^{k} \Phi\left(d_{1}-k \Sigma\right)\right)
\end{aligned}
$$

where

$$
d_{1,2}=\frac{\log \left(\frac{S}{J}\right)}{\Sigma} \pm \frac{1}{2} \Sigma, \quad \Sigma \equiv \sigma \sqrt{\tau} .
$$

From this, we find the following delta's

$$
\begin{aligned}
& \partial_{S} V_{L P}=-\Phi\left(-d_{1}\right)+k^{-1} \Phi\left(d_{1}\right)+\left(1-k^{-1}\right) e^{-\gamma \tau}\left(\frac{e^{\gamma \tau} J}{S}\right)^{k} \Phi\left(d_{1}-k \Sigma\right) \\
& \partial_{J} V_{L P}=\Phi\left(-d_{2}\right)-\left(\frac{e^{\gamma \tau} J}{S}\right)^{k-1} \Phi\left(d_{1}-k \Sigma\right) .
\end{aligned}
$$

In a very similar way, we can derive the value of the floating strike lookback call option. If we define $J=S_{\min } P$, its value is given by

$$
V_{L C}(S, J, \gamma, \tau)=V_{C}(S, J, \tau)+\frac{1}{k} V_{C}\left(R_{1-k}(S, J, \tau), S, k^{2} \tau\right)
$$

\section{Discussion and Outlook}

We have shown in this paper that the formalism put forward in Ref. 6 provides a powerful framework for the pricing of path-dependent contingent claim pricing. The formulation of the pricing problem in terms of tradables leads to more transparent 
formulae with clearer financial interpretations. The scale symmetry which should be satisfied at any time by the claim prices provides a very powerful check when doing computations. Exploiting symmetries of the governing PDE leads to large families of related claims. Also put-call symmetries follow naturally from the formalism.

In a first followup paper [7] we will discuss the pricing of arithmetic Asian options in greater detail. We will discuss intimate relations between the prices of unseasoned average price and average strike options, which follow from the local scale invariance. Furthermore the symmetry can be used to relate vanilla options on cash-dividend paying stock to arithmetic Asians. A second followup paper will discuss the benefits of using our symmetric formulation to compute prices of contingent claims numerically.

By formulating the pricing of claims in terms of tradables, we can also clarify and extend results on American-type options and stochastic volatility models. This will be discussed in future papers.

\section{Acknowledgments}

This work was carried out under the CWI-project MAS3.1 "Mathematical finance". The research of C. D. D. Neumann was partially supported by the SWON-program "Financial derivatives".

\section{References}

[1] M. Broadie, P. Glasserman and S. Kou, Connecting discrete and continous pathdependent options, Finance \& Stochastics 3(1) (1999) 55-82.

[2] P. Carr and M. Chesney, American put call symmetry, Working paper (1996).

[3] P. Carr and A. Chou, Hedging complex barrier options, Working paper (1997).

[4] B. Goldman, H. Sosin, and M. A. Gatto, Path-dependent options: Buy at the low, sell at the high, J. Finance 34 (1979) 1111-1127.

[5] H. Geman and M. Yor, Bessel processes, asian options an perpetuities, Mathematical Finance 3 (1993) 349-375.

[6] J. K. Hoogland and C. D. D. Neumann, Local scale invariance and contingent claim pricing, IJTAF 4(1) (2001) 1-21.

[7] J. K. Hoogland and C. D. D. Neumann, Asians and cash dividends: Exploiting symmetries in pricing theory, CWI Technical Report MAS-R0019 (2000).

[8] J. K. Hoogland and C. D. D. Neumann, Work in progress (2000).

[9] N. Kunitomo and M. Ikeda, Pricing options with curved boundaries, Mathematical Finance 2 (1992) 275-298.

[10] A. Kemna and A. Vorst, A pricing method for options based on average asset values, Journal of Banking and Finance 14 (1990) 113-129.

[11] N. Taleb, Dynamics hedging: Managing vanilla and exotic options, Wiley (1996).

[12] P. Wilmott, Derivatives, Wiley (1998). 